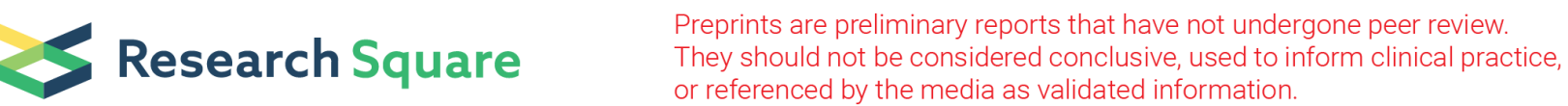

\section{Cross-cultural Adaptation and Validation of the Lebanese Arabic version of the BACS scale (the Brief Assessment of Cognition in Schizophrenia) among stable schizophrenic inpatients}

Chadia Haddad ( $\square$ Chadia_9@hotmail.com )

Psychiatric Hospital of the Cross: Hopital Psychiatrique de la Croix https://orcid.org/0000-0003-24132684

Pascale Salameh

Lebanese University: Universite Libanaise

Souheil Hallit

USEK: Universite Saint-Esprit de Kaslik

\section{Sahar Obeid}

USEK: Universite Saint-Esprit de Kaslik

\section{Georges Haddad}

Psychiatric Hospital of the Cross: Hopital Psychiatrique de la Croix

\section{Jean-Pierre Clément}

$\mathrm{CH}$ Esquirol: Centre Hospitalier Esquirol

\section{Benjamin Calvet}

CH Esquirol: Centre Hospitalier Esquirol

Research article

Keywords: Schizophrenia, Arabic, cognition, assessment, validation, BACS

Posted Date: February 10th, 2021

DOI: https://doi.org/10.21203/rs.3.rs-202703/v1

License: (c) (i) This work is licensed under a Creative Commons Attribution 4.0 International License.

Read Full License

Version of Record: A version of this preprint was published at European Psychiatry on April 1st, 2021. See the published version at https://doi.org/10.1192/j.eurpsy.2021.1405. 


\section{Abstract}

\section{Introduction}

Assessment of cognitive disorders in schizophrenia is becoming a part of clinical and research practice by using batteries that differ widely in their content. The Brief Assessment of Cognition in Schizophrenia (BACS) was developed to cover the main cognitive deficits of schizophrenia. The objective of this study was to assess concurrent validity of the Arabic version of the BACS with a standard neurocognitive battery of tests in Lebanese patients with schizophrenia and healthy controls.

\section{Methods}

A sample of 120 stable inpatients diagnosed with schizophrenia and 60 healthy controls received the Arabic version of the BACS in a first session, and a standard battery in a second session.

\section{Results}

Mean duration of completion for the BACS was $31.2 \pm 5.4 \mathrm{~min}$ in patients with schizophrenia. All tests demonstrated significant differences between controls and patients $(p<.01)$. Principal components analysis demonstrated that a one-factor solution best fits our dataset ( $64.8 \%$ of the variance). High Cronbach alpha was found (.85). The BACS composite scores were significantly correlated with the standard battery composite scores in patients $(r=.78, p<.001)$ and healthy controls $(r=.77, p<.001)$. Also, correlation analysis between the BACS sub-scores and the standard battery sub-scores showed significant results $(p<.05)$. The Arabic-BACS demonstrated high ability to discriminate patients with schizophrenia from healthy controls.

\section{Conclusion}

Results showed that the Arabic version of the BACS is a useful tool for assessing cognition in patients with schizophrenia and could be used in clinical practice in Lebanon.

\section{Introduction}

Patients with schizophrenia are characterized by deficits in a wide range of cognitive functions such as verbal memory, working memory, motor speed, attention, executive functions and verbal fluency that affect up to $75 \%$ of patients [1]. Cognitive function of patients with schizophrenia is lower by 1.5 to 2 SD (standard deviation) on several dimensions compared with results of healthy controls [2]. These cognitive impairments seemed to be independent of positive and negative symptoms and psychotic treatment, and are related to social and functional outcomes $[3,4]$. Therefore, cognitive assessment in patients with schizophrenia remains one of the best indicators of the functional and social prognosis of individuals.

Numerous neurocognitive batteries had been developed to assess cognitive impairment in patients with schizophrenia [5]. For example, the MATRICS (Measurement and Treatment Research to Improve 
Cognition in Schizophrenia) [6], the RBANS (Repeatable Battery for the Assessment of Neuropsychological Status) [7] and the CANTAB (Cambridge Neuropsychological Test Automated Battery) batteries [8]. However, most of them are long and complex, assess the entire neuropsychological profile of patients and may require several hours to be administered [9]. The availability of a brief and efficient tool for cognitive assessment in patients with schizophrenia could help clinicians making decisions about potential rehabilitation and adaptation of an appropriate antipsychotic drug treatments, as well as helping researchers assessing cognitive changes during clinical trials.

The Brief Assessment of Cognition in Schizophrenia (BACS) is a reliable and efficient test battery that assesses the main cognitive domains affected in schizophrenia such as verbal memory, working memory, speed of information processing, motor speed, verbal fluency and executive functions [10]. The BACS was designed to be easily administered by medical professionals such as psychiatric nurses, clinicians, psychiatrists, social workers, psychologists and other mental health professionals [10]. The duration of the test session is approximately 35 minutes with minimal extra time for scoring [10] (compared to more than two hours for a standard cognitive battery). The original version of the BACS was validated on a sample of 150 patients with schizophrenia compared to a sample of 50 healthy controls and had shown a high test-retest reliability (intraclass correlation coefficient $\geq 0.79$ ), sensitivity and validity in comparison to a standard battery of cognitive tests [10]: the global score on the BACS was highly correlated with that on the standard battery for patients $(r=0.79)$ as well as for controls $(r=0.90)$ [10]. The BACS has been translated in over 30 languages and validated in several of these including English [10], French [11], German [12], Spanish [13], Brazilian [14], Chinese [15], Japanese [16], Persian [17] and Italian [18]. These versions have shown acceptable reliability and concurrent validity with a standard cognitive battery.

Few studies have been done in the Arab countries that evaluate the cognitive impairment in patients with schizophrenia [19-21]. In Lebanon, some studies evaluated cognitive function in older people [22-24]. However, no study was done to assess cognitive functions in individuals with neurological or psychiatric diseases. Clinicians had limited options, using untranslated tests or using translations that have not been validated. Some neuropsychological batteries had been used locally among patients with schizophrenia to assess their cognition such as the Wechsler Adult Intelligence Scale (WAIS) and the RBANS. Therefore, the adaptation and validation of the BACS into Arabic would help researchers in assessing cognitive impairment in schizophrenia and guide clinical decisions on cognitive interventions and rehabilitation. Thus, the objective of this study is to assess concurrent validity of the Arabic version of the BACS with a standard neurocognitive battery of tests in Lebanese patients with schizophrenia and healthy controls.

\section{Methods}

\section{Study design and participants}

A cross-sectional study was conducted at the Psychiatric Hospital of the Cross - Lebanon (HPC), between July 2019 and Mars 2020. The study enrolled 120 inpatients diagnosed with schizophrenia and 
schizoaffective disorders and 60 healthy controls, matched for age, education and sex. The inclusion criteria for patients were as follows: inpatients aged between 18-60 years; having an educational level over five years, meeting the DSM-5 criteria (Diagnostic and Statistical Manual of Mental Disorders, fifth edition) for schizophrenia and schizoaffective disorders; receiving antipsychotic medication and clinically stable. There were no specific medication criteria for inclusion in the patient group. The healthy individuals were recruited from the staff of the HPC hospital and meeting the following criterion: absence of a history of major psychiatric disorders. Exclusion criteria for all participants included brain trauma, neurological disorder or current substance use disorder that would influence cognitive performance.

Based on a list generated from the hospital's computer software, out of 180 patients selected according to the inclusion criteria, 120 patients (71 males and 49 females) were included. Sixty patients were excluded (40 males and 20 females) for the following reasons: 22 patients refused to participate, 21 left the hospital, 13 refused to continue the assessment and 4 have a difficulty of performing the cognitive tests (Figure 1). Those who agreed to participate in the study were requested to sign a written informed consent form without any monetary compensation.

\section{Sample size calculation}

Using the Gpower 3.1.9.2 software for the calculation of the minimal sample size needed for our study, with a power of $80 \%(1-\beta=0.8)$ and an error a of 0.05 , an effect size of 0.47 was calculated based on the original study done by Keefe et al [10]. In this study the BACS battery composite score was highly correlated with the standard battery composite scores in patients with schizophrenia $(r=0.76)$ and healthy controls $(r=0.90)$. Considering a ratio of $2: 1$ in each group, the results showed that the minimal sample size needed was 164 (55 in the healthy control group and 109 in the patient group).

\section{Adaptation and assessment procedure}

The Arabic version of the BACS has already been translated from English into classical Arabic language by Ateeq and collaborators using the standard translation/back-translation procedure among 33 patients with schizophrenia from Riyadh city (unpublished study). This Arabic version was sent to us by Pr. Richard Keefe and was checked and approved by the Department of Psychiatry and Behavioral Sciences at the University of Duke Medical Center.

Demographic variables (age, gender, marital status, educational level and monthly income) and clinical information of the participants (diagnosis, duration of illness in years, duration of hospitalization in years, number of hospitalization, medication intakes, and family history of mental disorders) were collected from medical files. The socioeconomic status was divided into four levels (no income, low (< 1.000 USD), intermediate (1.000-2.000 USD), high (>2.000USD)) and the education level was divided into three levels (complementary level ( $>5$ years), secondary level ( $>9$ years) and university level $(>12$ years).

Participants were tested on two separate days by two independent psychologists (one trained in the administration of the BACS and the other one trained in use of the standard tests battery) with less than 
two weeks between the two assessments. There were two versions of the BACS (version A and version B). These versions have the same tests with alternate forms. In our study we used version A. For the first test session, subjects received version A of the BACS and for the second session, the standard battery of cognitive tests was administered.

The BACS, included the tests listed below in the administered order:

Verbal memory - list learning

Subjects were presented with 15 words and then asked to recall as many words as possible. This procedure was repeated five times. Performance was measured as number of words recalled per trial, in any order (with a range between 0 and 75$)$.

\section{Working memory - digit sequencing task}

Subjects were presented with clusters of numbers of increasing length. They were asked to tell the experimenter the numbers in order, from lowest to highest. Performance was measured as number of correct responses (with a range between 0 and 28).

\section{Motor speed - token motor task}

Subjects were given 100 plastic tokens and asked to place them two at a time into a container as quickly as possible. A 60-s time limit was imposed. Performance was measured in terms of the number of tokens correctly placed into the container for the first half-minute, second half-minute and the 1-min total (with a range between 0 and 100 at the final outcome).

\section{Verbal fluency}

Category instances. Subjects were given 60 seconds to name as many words as possible within a given category (animals).

Controlled oral words association test. In two separate trials, subjects were given 60 seconds to generate as many words as possible that began with a given letter: T, R. The letters " $\square$ " (similar to M in English) and letter "ᄆ" (similar to G in English) were used since these letters in Arabic had as much word redundancy as the letters $\mathrm{T}$ and $\mathrm{R}$ in English. The overall test score refers to the number of words generated correctly within 60 seconds. The total score for the verbal fluency test refers to the sum of the three trials. Higher scores reflect a better performance.

\section{Attention and speed information processing - symbol coding}

Subjects were required to write as quickly as possible the numerals 1-9 as matches to symbols on a response sheet for 90 seconds. The measure of performance consisted in the number of correct numerals (with a range between 0 and 110). 
Subjects were shown two pictures simultaneously. Each picture showed three balls of different colors arranged on three pegs, with the balls in a unique arrangement in each picture. Subjects were asked to give the total number of times the balls in one picture needed to be moved in order to make the arrangement of balls identical to that of the other, opposing picture. There were 20 trials. The items were of variable difficulty, with a general tendency for later items to be more difficult. If patients responded correctly to all 20 trials, two additional trials of greater difficulty were administered. The measure of performance consisted in the number of correct responses (with a range between 0 and 22).

\section{Standard battery}

The standard battery consisted of tests designed to examine the same constructs as the BACS. In the order given, the tests and their respective constructs are listed as follow: 16 item Free and Cued Recall test (RL/RI-16) (verbal memory), Forward and Backward Digit Span Sequencing from the WAIS-IV (working memory) [25], Trail Making Test A (TMT-A) (motor speed), Controlled Oral Word Association Test (letter "[" (similar to B in English) and "ᄆ" (similar to F in English), Category Instances (Fruit category) (verbal fluency), Digit Symbol Coding from the WAIS-IV (attention and speed of information processing) [25] and Block Design Test from the WAIS-IV (reasoning and problem solving) [25].

\section{Data analysis}

Data analysis was done using the SPSS software version 25. To check the distribution of normality for the BACS scale, we used the Shapiro Wilk test and we found that the major dependent variable was normally distributed. A descriptive analysis was performed where categorical variables were expressed as absolute frequencies and percentages and quantitative variables as means and standard deviations. The Chi-square and Fisher exact tests were used to compare between categorical variables and patients and controls groups while the Student T-test was used to compare continuous variables between groups.

The Arabic-BACS composite scores and the standard neurocognitive battery were determined by averaging all the subscales of each instrument, after transforming them to z-scores. Concurrent validity of the Arabic-BACS subscales was tested by using Pearson correlations with the corresponding scales of the standard battery. Construct validity of the BACS scores was assessed using the principal component analysis. To ensure the model's adequacy, Kaiser-Meyer-Olkin measure of sampling adequacy and Bartlett's test of sphericity were calculated. Factors with eigenvalues values larger than one were retained and scree plot method was used for determining the number of components to extract [26]. Only items with factor loading larger than 0.4 were considered [27]. Moreover, internal consistency of the ArabicBACS was assessed using the Cronbach's alpha. Face validity was examined by comparing subtest scores between patients and controls through Student T-test. Threshold for discrimination between schizophrenic cases and controls was determined, in addition to sensitivity and specificity, using receiver-operator characteristics (ROC) curves, where all schizophrenic patients were considered "cases" and all controls "non-cases". P-values less than 0.05 were considered statistically significant. 


\section{Results}

\section{Sociodemographic characteristics}

Sociodemographic characteristics of the participants are summarized in Table 1. Mean age of patients with schizophrenia was $48.4 \pm 7.6$ years, with $59.2 \%$ males. The majority $(81.9 \%)$ were single, with low monthly income $(52.6 \%), 50 \%$ have a secondary level of education and $36.5 \%$ have a family history of psychiatric illness. Mean duration of illness and hospitalization were $20.6 \pm 12.4$ and $12.4 \pm 8.5$ years respectively. The healthy control group was matched with the schizophrenia group according to gender, education level and age. The two groups differ on marital status, monthly income and family history of psychiatric illness. Married participants with high monthly income and without any psychiatric illness were found in the control group as compared to the patient group. 
Table 1: Sociodemographic characteristics of the sample

Patients with Schizophrenia
$(\mathrm{N}=120)$

Frequency (\%)

\section{Healthy}

controls

$(\mathrm{N}=60)$

Frequency (\%)

\section{Gender}

Male

$71(59.2 \%)$

$36(60.0 \%)$

0.91

Female

$49(40.8 \%)$

$24(40.0 \%)$

\section{Education level}

Complementary

$41(34.2 \%)$

$21(35.0 \%)$

0.73

Secondary

$60(50.0 \%)$

27 (45.0\%)

University

19 (15.8\%)

$12(20.0 \%)$

\section{Marital Status}

Single

95 (81.9\%)

$6(10.0 \%)$ $<0.001$

Married

$9(7.8 \%)$

52 (86.7\%)

Widowed

$2(1.7 \%)$

$0(0.0 \%)$

Divorced

10 (8.6\%)

$2(3.3 \%)$

Monthly income

No income

27 (23.3\%)

$0(0.0 \%)$

$<0.001$

$<1000$ \$

61 (52.6\%)

$39(67.2 \%)$

$1000-2000 \$$

$26(22.4 \%)$

$12(20.7 \%)$

$>2000 \$$

$2(1.7 \%)$

$7(12.1 \%)$

Family history of psychiatric illness

\begin{tabular}{llll} 
Yes & $42(36.5 \%)$ & $5(8.5 \%)$ & $<0.001$ \\
\cline { 1 - 3 } No & $73(63.5 \%)$ & $54(91.5 \%)$ & \\
\hline Mean \pm SD & $48.4 \pm 7.6$ & Mean \pm SD & \\
\hline Age & $20.6 \pm 12.4$ & - & 0.67 \\
\hline $\begin{array}{l}\text { Duration of illness (years) } \\
\text { (years) }\end{array}$ & $12.4 \pm 8.5$ & - & \\
\hline
\end{tabular}




\section{Testing duration}

The BACS required a mean of $31.2 \pm 5.4 \mathrm{~min}$ for patients and $30.1 \pm 3.1 \mathrm{~min}$ for healthy controls. The standard battery required a mean of $42.3 \pm 10.6 \mathrm{~min}$ for patients and $35.9 \pm 3.7 \mathrm{~min}$ for healthy controls.

\section{Comparison of the mean BACS measures between patients with schizophrenia and healthy controls}

Table 2 presents performances of schizophrenia patients and controls on the standard battery and BACS battery tests, including group means and standard deviations for measures of each test and z-scores for patients. Significantly lower means of standard battery subtests and total score were found in patients with schizophrenia as compared to healthy control ( $p<.001$ for all). Also, a significant difference was found in the mean BACS measures between the two groups with a lower mean in all the BACS subtests and total score inpatients with schizophrenia compared to healthy controls $(p<0.001$ for all). 
Table 2: Performances of patients with schizophrenia and healthy controls on standard battery and BACS battery tests

\begin{tabular}{|c|c|c|c|c|}
\hline & \multicolumn{2}{|l|}{$\begin{array}{l}\text { Patients with Schizophrenia } \\
(\mathrm{N}=120)\end{array}$} & \multirow{2}{*}{$\begin{array}{l}\text { Healthy } \\
\text { control } \\
(\mathrm{N}=60)\end{array}$} & \multirow[t]{3}{*}{$\begin{array}{l}P \\
\text { value }\end{array}$} \\
\hline & & & & \\
\hline & Mean \pm SD & $\begin{array}{l}Z \\
\text { score }\end{array}$ & Mean \pm SD & \\
\hline Standard battery test & $241.8 \pm 125.2$ & -3.7 & $432.0 \pm 52.0$ & $<.001$ \\
\hline $\begin{array}{l}\text { Free and Cued Recall test (RL/RI- } \\
\text { 16) }\end{array}$ & $25.9 \pm 14.8$ & -2.2 & $44.3 \pm 8.3$ & $<.001$ \\
\hline $\begin{array}{l}\text { Digit span sequencing from the } \\
\text { WAIS-IV }\end{array}$ & $10.0 \pm 3.4$ & -0.9 & $13.4 \pm 3.6$ & $<.001$ \\
\hline The Trail Making Test A (TMT-A) & $150.7 \pm 91.4$ & -4.6 & $48.9 \pm 22.1$ & $<$. \\
\hline Verbal Fluency test & $19.6 \pm 8.5$ & -2.1 & $36.4 \pm 8.1$ & $<.001$ \\
\hline Fruit category & $11.1 \pm 4.6$ & -2.3 & $21.5 \pm 4.6$ & $<.001$ \\
\hline Letter B & $4.7 \pm 2.7$ & -1.2 & $8.2 \pm 2.9$ & $<.001$ \\
\hline Letter F & $3.8 \pm 2.6$ & -0.9 & $6.7 \pm 3.1$ & $<.001$ \\
\hline $\begin{array}{l}\text { Digit Symbol Coding from the } \\
\text { WAIS-IV }\end{array}$ & $19.2 \pm 14.2$ & -2.0 & $54.3 \pm 17.4$ & $<.001$ \\
\hline $\begin{array}{l}\text { Block design test from the WAIS- } \\
\text { IV }\end{array}$ & $17.9 \pm 11.5$ & -1.4 & $32.5 \pm 10.8$ & $<.001$ \\
\hline BACS Battery test & $109.9 \pm 47.2$ & -2.9 & $221.7 \pm 38.5$ & $<.001$ \\
\hline List learning test & $20.9 \pm 9.6$ & -2.1 & $41.1 \pm 9.4$ & $<.001$ \\
\hline Digit sequencing & $10.6 \pm 5.5$ & -1.9 & $19.3 \pm 4.4$ & $<.001$ \\
\hline Token motor task & $35.7 \pm 15.0$ & -2.4 & $70.7 \pm 14.4$ & $<.001$ \\
\hline Verbal fluency & $20.4 \pm 9.2$ & -1.5 & $34.6 \pm 9.2$ & $<.001$ \\
\hline Animal category & $11.5 \pm 4.7$ & -1.6 & $18.1 \pm 4.1$ & $<.001$ \\
\hline
\end{tabular}




\begin{tabular}{|lcccc|}
\hline Letter G & $4.2 \pm 2.7$ & -1.0 & $7.4 \pm 3.2$ & $<$ \\
\hline Letter M & $4.7 \pm 3.4$ & -1.1 & $9.1 \pm 3.9$ & $<$ \\
& & & & .001 \\
\hline Symbol coding & $12.2 \pm 12.4$ & -2.4 & $38.0 \pm 10.7$ & $<$ \\
& $9.9 \pm 7.7$ & -2.1 & $17.9 \pm 3.7$ & $<$ \\
\hline Tower of London & & & & .001 \\
\hline
\end{tabular}

\section{BACS composite score profile}

Figure 2 shows mean composite scores for the BACS total score and subtests and standard battery in patients with schizophrenia compared to healthy controls. All differences between patients and controls were statistically significant $(p<.001)$. Motor speed $(z=-2.43)$ was the most deficient function. The following was attention and speed information processing ( $z=-2.39)$.Also, significant differences were found between the mean composite scores from the BACS and the standard battery $(-2.9$ vs. $-3.7 ; p$ $<.001)$.

\section{Correlations among BACS measures}

Table 3 presents correlations among BACS measures for patients and healthy controls. Among patients with schizophrenia, all correlations were highly significant $(p<0.01)$. Among healthy controls, all correlations were significant except correlation between token motor and tower of London. 
Table 3: Correlations among BACS measures for schizophrenia patients and healthy controls

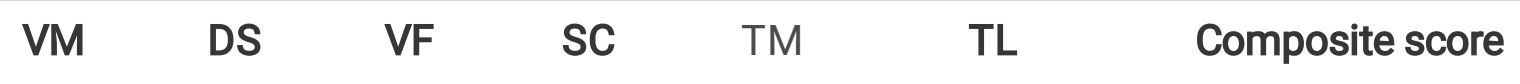

In patients with schizophrenia

\begin{tabular}{lccccccc} 
TL & .54 & .72 & .57 & .58 & .52 & - & .79 \\
\hline TM & .49 & .54 & .55 & .46 & - & .52 & .79 \\
SC & .50 & .61 & .57 & - & .46 & .58 & .79 \\
VF & .65 & .72 & - & .57 & .55 & .57 & .83 \\
DS & .60 & - & .72 & .61 & .54 & .72 & .83 \\
VM & - & .60 & .65 & .50 & .49 & .54 & .77
\end{tabular}

In healthy controls

\begin{tabular}{|lccccccc} 
TL & .35 & .41 & .37 & .43 & .05 & - & .45 \\
TM & .41 & .31 & .37 & .38 & - & .05 & .71 \\
\hline SC & .61 & .61 & .61 & - & .38 & .43 & .82 \\
VF & .58 & .56 & - & .61 & .37 & .37 & .79 \\
DS & .48 & - & .56 & .61 & .31 & .41 & .69 \\
VM & - & .48 & .58 & .61 & .41 & .35 & .80
\end{tabular}

Among patients, all correlations were significant (less than 0.001 , greater than or equal to $0.46, \mathrm{P}<$ 0.001 ). Among controls, correlations greater than or equal to $0.47, \mathrm{P}<0.001 ; 0.34-0.46, \mathrm{P}<0.01$; $0.34-0.31, P<0.05$, correlation equal to $0.05, p=0.701$.

VM: Verbal Memory; DS: Digit sequencing; VF: Verbal Fluency; SC: Symbol Coding; TM: Token motor task; TL: Tower of London.

\section{Factor analysis of the BACS}

Factor analysis for the BACS test was run over the schizophrenia sample. All items could be extracted from the list, but none of the items was removed because no item over-correlated to each other $(r>0.9)$, had a low loading on factors $(<0.3)$ or a low communality $(<0.3)$. BACS subtest items converged over a one factor that had an eigenvalue over 1, explaining a total of $64.8 \%$ of the variance. Kaiser-Meyer-Olkin measure of sampling adequacy of 0.885 was found, with a significant Bartlett's test of sphericity $(p<$ 0.001). According to the promax rotated matrix, components are summarized in Table 4. Moreover, a high Cronbach's alpha was found for the full test (0.85). 


\begin{tabular}{|lc|}
\hline \multicolumn{2}{|l|}{ Table 4: Factor loading of BACS measures in patients with schizophrenia } \\
\hline Digit sequencing & Factor $\mathbf{1}$ \\
\hline Verbal Fluency & .88 \\
\hline Tower of London & .85 \\
\hline Verbal Memory & .82 \\
\hline Symbol Coding & .78 \\
\hline Token motor task & .77 \\
\hline Cronbach's alpha & .73 \\
\hline Percentage of variances explained & .85 \\
\hline
\end{tabular}

\section{Correlations between standard battery and BACS measures}

Correlations between standard battery constructs and BACS measures are presented in Table 5. In each matrix, correlations were similar between the two groups (patients with schizophrenia and healthy controls). Correlations between standard battery and BACS composite scores were 0.77 and 0.78 in patient group and control group respectively. Individual data points from this correlation are presented in Figure 3. 
Table 5: Pearson correlations between standard battery domains and BACS measures

Standard Battery

BACS measures

VM DS TM VF SC TL Composite BACS

score

\section{In patients with schizophrenia}

Free and Cued Recall test (RL/RI-16)

$\begin{array}{lllllll}.57 & .61 & .28 & .61 & .46 & .49 & .59\end{array}$

Digit span sequencing from the WAIS-IV

$\begin{array}{lllllll}.44 & .75 & .42 & .68 & .65 & .58 & .70\end{array}$

The Trail Making Test A (TMT-A)

$\begin{array}{lllllll}.44 & .60 & .51 & .55 & .59 & .46 & .66\end{array}$

The Controlled Oral Word Association Test;

$\begin{array}{lll}.62 & .68 & .45\end{array}$

$\begin{array}{llll}.82 & .56 & .51 & .74\end{array}$

Category Instances

Digit Symbol Coding from the WAIS-IV

$\begin{array}{lllllll}.54 & .60 & .53 & .57 & .84 & .51 & .76\end{array}$

Block design test from the WAIS-IV

$\begin{array}{lllllll}.53 & .60 & .42 & .55 & .53 & .63 & .66\end{array}$

Composite standard battery score

.55

In healthy control

Free and Cued Recall test (RL/RI-16)

$\begin{array}{lllllll}.64 & .35 & .22 & .53 & .54 & .26 & .58\end{array}$

Digit span sequencing from the WAIS-IV

$\begin{array}{lllllll}.48 & .54 & .35 & .42 & .61 & .24 & .60\end{array}$

The Trail Making Test A (TMT-A)

$\begin{array}{lllllll}.50 & .50 & .21 & .50 & .58 & .58 & .60\end{array}$

The Controlled Oral Word Association Test;

Category Instances

$\begin{array}{lllllll}.53 & .55 & .34 & .73 & .52 & .26 & .66\end{array}$

Digit Symbol Coding from the WAIS-IV

$\begin{array}{lllllll}.57 & .51 & .28 & .43 & .83 & .28 & .67\end{array}$

Block design test from the WAIS-IV

$\begin{array}{lllllll}.27 & .37 & .11 & .23 & .38 & .34 & .34\end{array}$

Composite standard battery score

$\begin{array}{lll}.68 \quad .64 & .32\end{array}$

$\begin{array}{llll}.63 & .81 & .51 & .78\end{array}$

Among patients, all correlations were significant less than 0.001 except correlation between TM and Free and Cued Recall test (RL/RI-16) equal to $0.28, p=0.002$.

Among controls, correlations greater than or equal to $0.49, \mathrm{P}<0.001 ; 0.33-0.48, \mathrm{P}<0.01 ; 0.28-0.32, \mathrm{P}<$ $0.05 ; 0.27-0.11 p>0.05$.

VM: Verbal Memory; DS: Digit Sequencing; TM: Token Motor Task; VF: Verbal Fluency; SC: Symbol Coding; TL: Tower of London.

\section{ROC analysis}


In order to examine the contribution of the BACS composite score for differentiating patients with schizophrenia from healthy controls, a ROC analysis was conducted. The area under the ROC was .96 (confidence interval $=.94-99, P \leq .001$ ), sensitivity was .93 and specificity was .86 with the cut-off value of 163 ( $z$ score $=-1.51)$. This result indicates an excellent ability of composite score of the BACS to discriminate between schizophrenia patients and controls (Fig. 3).

\section{Discussion}

The Lebanese Arabic version of the BACS showed a good concurrent validity with a standard cognitive battery of tests, high internal consistency with one factor structure and an ability to differentiate patients with schizophrenia disorders from healthy controls. Time required to administer BACS in patients was $31.2 \mathrm{~min}$ almost equal to the original BACS where the completed time was $34.2 \mathrm{~min}$ [10]. Testing period could be shortened by becoming more familiar with the administration of tests. Administration durations of the BACS in patients and controls were similar displaying a consistent performance of the administrator. Duration difference between administration of BACS (31.18 min) and of standard battery (42.33 $\mathrm{min}$ ) in patients was $11.15 \mathrm{~min}$, which could be explained by the longer duration required by some standard battery tests (for example RL/RI-16 test).

Good concurrent validity was found between BACS and standard battery composite scores $(r=0.76)$ similar to correlations found in the original BACS article [10]. BACS is a good neurocognitive battery for assessing global cognitive function in patients with schizophrenia. Concerning subtests of the BACS, we also found a good correlation ( $r>0.70)$ for digit sequencing, symbol coding and verbal fluency but less good for tower of London $(r=0.63)$. Lower correlations were found for verbal memory $(r=0.57)$ and motor speed tests $(r=0.51)$. Similar results were found for the motor speed tests in the original version [10], French [11], German [12], Persian [17] and Spanish [13] versions. Low correlations between Token Motor Task and TMT A could be explained by the fact that these tasks assess slightly different aspects of cognitive functions [17]. For the verbal memory task, a methodological difference could exist in assessment of episodic memory between list learning test and RL/RI-16 tests [28]. Also, these two tests had not yet been adapted and validated into the Lebanese language, as alternate words might facilitate the assessment as difficulty in remembering the required words might exist.

Factor analysis performed on the Arabic-BACS showed a one-factor solution underlying the BACS subtests, which explained $64.8 \%$ of the instrument total variance. Similarly, a unique factor structure was found in the Spanish [13] and Persian versions [17]. While the original BACS [10] and the Japanese BACS [16] found a three factor solution, and the French BACS [11] showed a two factor solution, they all found that a single factor explained most of the variance. The discrepancies among the studies could be explained by different sample size and differences in clinical symptoms among participants. We also found a high degree of internal consistency revealed by an elevated Cronbach's alpha and by statistically significant correlations between individual items of the scale with Pearson correlation analysis. Our findings indicate that the BACS can be used to assess general cognitive performance in schizophrenia. 
Results of ROC analysis showed that BACS composite score at a level of -1.51 had high level of sensitivity and specificity to differentiate patients with schizophrenia and controls. In line with the German [12], Chinese [15] and Persian [17] versions, our result indicates that the Arabic-BACS is a useful tool for discriminating patients and controls based on neurocognitive function. All the subscales and total scale scores of the Arabic-BACS differed significantly between patients and controls. We also noted that mean BACS global score and subscales scores had high deficiency as compared to the original version [10]. Also, the cognitive task that was mostly deficient in our patients was the motor speed followed by attention and processing speed. However in the original article the most deficient cognitive task was verbal memory followed by attention and processing speed [10]. It is well recognized that psychomotor task is the first affected cognitive domain in schizophrenia [29]. Our results could be explained that selected patients were institutionalized for a long period and might have more cognitive impairment than outpatients selected from outpatient clinics.

\section{Limitation}

Several limitations have been found in this study. First, results of the study could not be generalized to the population because of the small sample size and patients were selected from one single site. Second, information bias might have occurred since accurate details could not be provided from participants in a face-to-face interview. Third, test retest was not assessed. Finally, further comparisons need to be made for the Verbal Memory task. A complete validation of the BACS will require further studies implementing assessments among specific schizophrenic sub-groups (first episode populations, treatment refractory schizophrenic patients and geriatric patients).

\section{Conclusion}

This Arabic version of the BACS showed satisfactory psychometric properties, including high internal consistency, acceptable concurrent validity, and good overall discriminant validity. We suggest that Arabic BACS is a reliable and practical tool for assessing cognitive function among inpatients with schizophrenia in daily psychiatry clinical practice and in research studies. However, it remains uncertain whether findings in this study could be generalized to Arabian populations who speak other dialects. Normative data in healthy population should be obtained in future for the BACS to meet all the requirements of a good cognitive instrument.

\section{Abbreviations}

BACS: Brief Assessment of Cognition in Schizophrenia, SD: Standard deviation, MATRICS: Measurement and Treatment Research to Improve Cognition in Schizophrenia, RBANS: Repeatable Battery for the Assessment of Neuropsychological Status, CANTAB: Cambridge Neuropsychological Test Automated Battery, WAIS: Wechsler Adult Intelligence Scale, HPC: Psychiatric Hospital of the Cross, DSM-5: Diagnostic and Statistical Manual of Mental Disorders, fifth edition, USD: United States dollar, TMT-A: Trail Making Test A, SPSS: Statistical Package for Social Sciences, VM: Verbal Memory, DS: Digit 
sequencing, VF: Verbal Fluency, SC: Symbol Coding, TM: Token motor task, TL: Tower of London, ROC: receiver operating characteristic curve.

\section{Declarations}

\section{Ethical Approval}

This study was approved by the Psychiatric Hospital of the Cross Ethics and Research Committee (HPC024-2018) in compliance with the Hospital's Regulatory Research Protocol. Each participant was informed of the purpose and requirement of the study. Consent was obtained as written approval on the ethical informed consent form. The procedures used in this study adhere to the tenets of the Declaration of Helsinki.

\section{Consent for publication}

Not applicable.

\section{Availability of data and materials}

Data can be made available under reasonable request form the corresponding author.

\section{Competing interests}

The authors have nothing to disclose.

\section{Funding}

None.

\section{Authors' contribution}

$\mathrm{BC}$, JPC designed the study; $\mathrm{CH}$ drafted the manuscript; $\mathrm{CH}$ carried out the analysis and interpreted the results; $\mathrm{BC}, \mathrm{JPC}, \mathrm{PS}, \mathrm{SH}, \mathrm{SA}, \mathrm{GH}$ assisted in drafting and reviewing the manuscript; $\mathrm{CH}$ was responsible for data collection; JPC, BC supervised the course of the article. All authors reviewed and approved the final version of the manuscript.

\section{Acknowledgments}

This work was supported by the Psychiatric hospital of the Cross - Lebanon (PHC), the Psychiatric hospital center in Limoges (CH Esquirol) and the UMR INSERM 1094 Limoges - France. The authors would like to thank Vanessa Mortada and Layale Hanna who helped in participants recruitment and thank all the individuals (patients and employees) who participated in this study. Also, the authors express their deepest gratitude to Professor Richard SE Keefe for providing scientific feedback and granting permission to use the Arabic version of the BACS. 


\section{References}

1. Millan, M.J., et al., Cognitive dysfunction in psychiatric disorders: characteristics, causes and the quest for improved therapy. Nature reviews Drug discovery, 2012. 11(2): p. 141-168.

2. Nuechterlein, K.H., et al., Identification of separable cognitive factors in schizophrenia. Schizophrenia research, 2004. 72(1): p. 29-39.

3. Keefe, R.S., et al., The relationship of the Brief Assessment of Cognition in Schizophrenia (BACS) to functional capacity and real-world functional outcome. Journal of Clinical and Experimental Neuropsychology, 2006. 28(2): p. 260-269.

4. McGurk, S.R. and K.T. Mueser, Cognitive functioning, symptoms, and work in supported employment: a review and heuristic model. Schizophrenia research, 2004. 70(2-3): p. 147-173.

5. Bakkour, N., et al., Systematic review of appropriate cognitive assessment instruments used in clinical trials of schizophrenia, major depressive disorder and bipolar disorder. Psychiatry research, 2014. 216(3): p. 291-302.

6. Nuechterlein, K.H. and M.F. Green, MATRICS consensus cognitive battery manual. Los Angeles, CA: MATRICS Assessment Inc, 2006.

7. Randolph, C., Repeatable Battery for the Assessment of Neuropsychological Status (RBANS). 1998: Psychological Corporation San Antonio.

8. Robbins, T., et al., A neural systems approach to the cognitive psychology of ageing using the CANTAB battery, in Methodology of frontal and executive function. 2004, Routledge. p. 216-239.

9. Reichenberg, A.A., The assessment of neuropsychological functioning in schizophrenia. Dialogues in clinical neuroscience, 2010. 12(3): p. 383.

10. Keefe, R.S., et al., The Brief Assessment of Cognition in Schizophrenia: reliability, sensitivity, and comparison with a standard neurocognitive battery. Schizophrenia research, 2004. 68(2-3): p. 283297.

11. Bralet, M.-C., et al., Validation of the French version of the BACS (the brief assessment of cognition in schizophrenia) among 50 French schizophrenic patients. European Psychiatry, 2007. 22(6): p. 365370 .

12. Sachs, G., et al., Validation of the German version of the Brief Assessment of Cognition in Schizophrenia (BACS)-preliminary results. European Psychiatry, 2011. 26(2): p. 74-77.

13. Segarra, N., et al., Spanish validation of the Brief Assessment in Cognition in Schizophrenia (BACS) in patients with schizophrenia and healthy controls. European Psychiatry, 2011. 26(2): p. 69-73.

14. Salgado, J.V., et al., Sensitivity and applicability of the Brazilian version of the Brief Assessment of Cognition in Schizophrenia (BACS). Dementia \& neuropsychologia, 2007. 1(3): p. 260.

15. Wang, L.-J., et al., Validation of the Chinese version of Brief Assessment of Cognition in Schizophrenia. Neuropsychiatric disease and treatment, 2016. 12: p. 2819.

16. Kaneda, Y., et al., Brief assessment of cognition in schizophrenia: validation of the Japanese version. Psychiatry and Clinical Neurosciences, 2007. 61(6): p. 602-609. 
17. Mazhari, S., et al., Validation of the P ersian version of the Brief Assessment of Cognition in Schizophrenia in patients with schizophrenia and healthy controls. Psychiatry and clinical neurosciences, 2014. 68(2): p. 160-166.

18. Anselmetti, S., et al., The brief assessment of cognition in schizophrenia. Normative data for the Italian population. Neurological Sciences, 2008. 29(2): p. 85-92.

19. El-Hadidy, M.A., W. Elemshaty, and W. Othman, Could Infection Effect Cognitive Function in Schizophrenia? One Egyptian Center Study. Dear Reader, 2013. 24(2): p. 85-92.

20. Gohar, S.M., et al., Adapting and evaluating a social cognitive remediation program for schizophrenia in Arabic. Schizophrenia research, 2013. 148(1-3): p. 12-17.

21. Ben, O.A., et al., The Tunisian cognitive battery for patients with schizophrenia. La Tunisie Medicale, 2009. 87(10): p. 674-679.

22. El Zoghbi, M., et al., Association between cognitive function and nutritional status in elderly: A crosssectional study in three institutions of Beirut-Lebanon. Geriatric Mental Health Care, 2013. 1(4): p. 73-81.

23. Bou-Orm, I.R., A.M. Khamis, and M. Chaaya, Determinants of poor cognitive function using A-IQCODE among Lebanese older adults: a cross-sectional study. Aging \& mental health, 2018. 22(6): p. 844848.

24. Abou-Mrad, F., et al., Screening for dementia in Arabic: normative data from an elderly Lebanese sample. The Clinical Neuropsychologist, 2017. 31(sup1): p. 1-19.

25. Hartman, D.E., Wechsler Adult Intelligence Scale IV (WAIS IV): return of the gold standard. Applied neuropsychology, 2009. 16(1): p. 85-87.

26. Kanyongo, G.Y., Determining the correct number of components to extract from a principal components analysis: A Monte Carlo study of the accuracy of the scree plot. Journal of Modern Applied Statistical Methods, 2005. 4(1): p. 13.

27. Ellis, J.L., Factor analysis and item analysis. Applying Statistics in Behavioural Research, 2017: p. 1159.

28. Drolet, V., et al., Comparaison des performances à l'épreuve des 15 mots de Rey et au RL/RI 16 dans le vieillissement normal et la démence de type Alzheimer. Gériatrie et Psychologie Neuropsychiatrie du Vieillissement, 2014. 12(2): p. 218-226.

29. Morrens, M., W. Hulstijn, and B. Sabbe, Psychomotor slowing in schizophrenia. Schizophrenia bulletin, 2007. 33(4): p. 1038-1053.

\section{Figures}




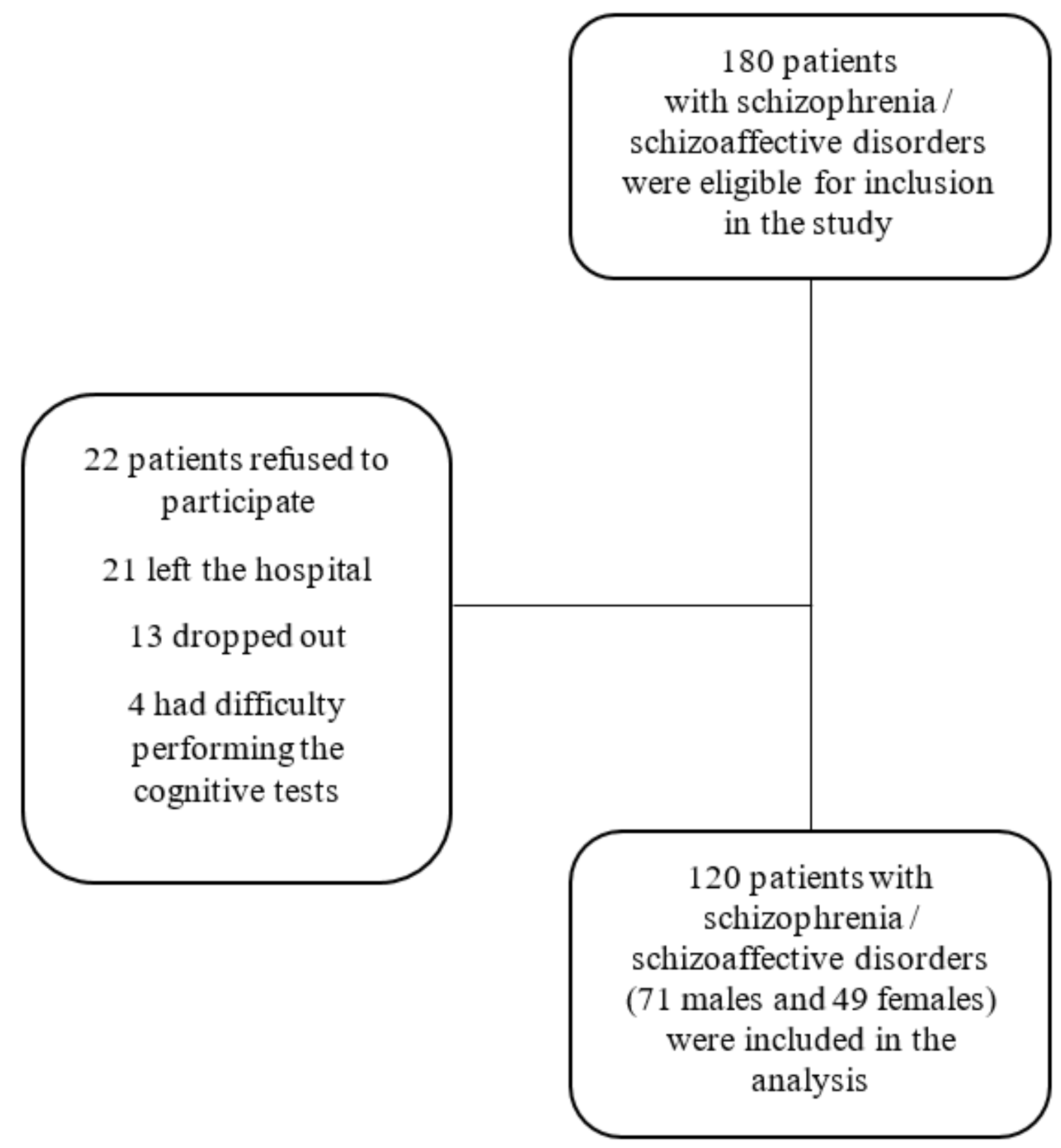

Figure 1

Enrollment of inpatients with schizophrenia. 


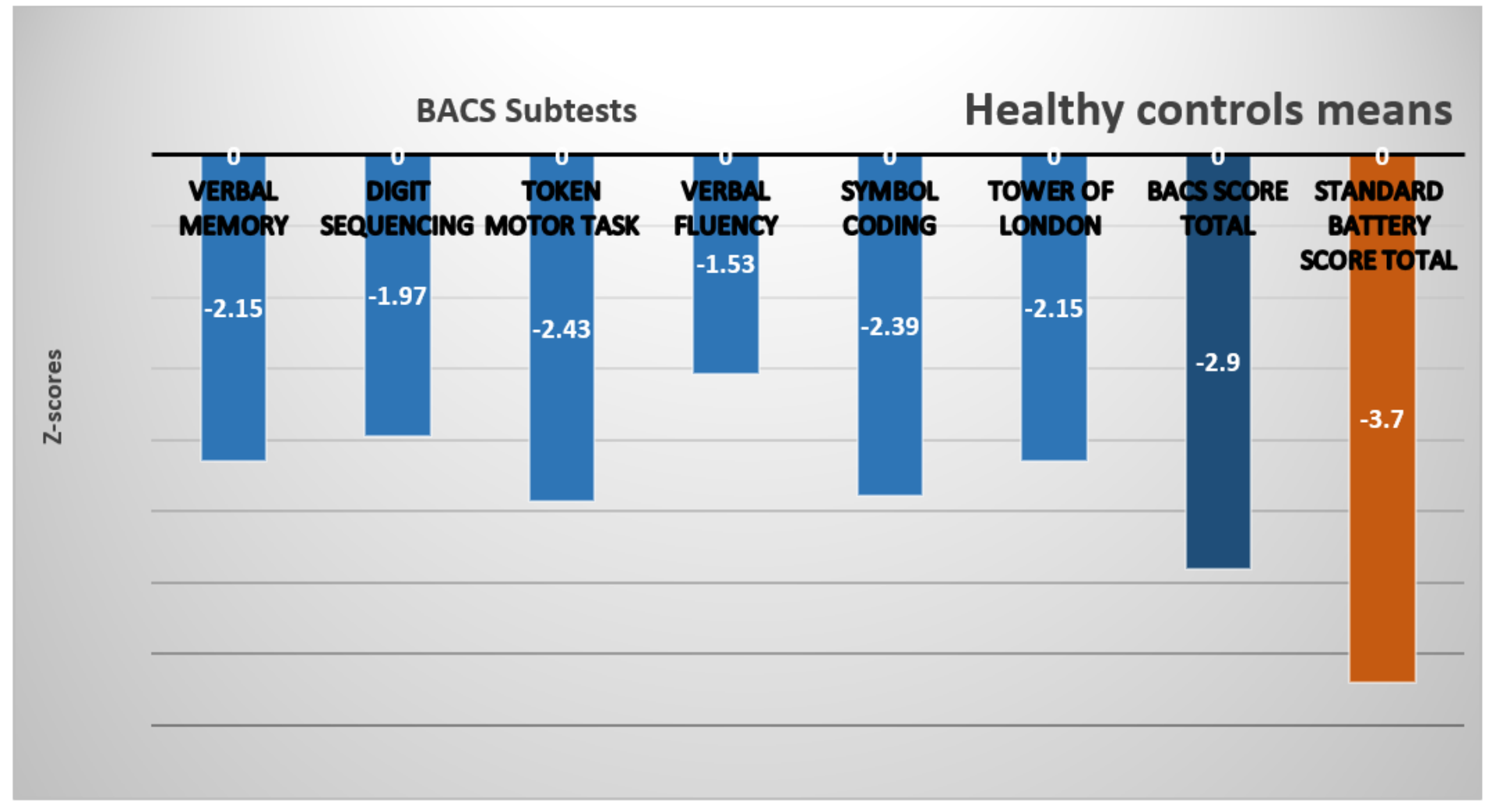

Figure 2

Composite scores for the BACS total score and subtests and standard battery in patients with schizophrenia standardized to healthy controls 


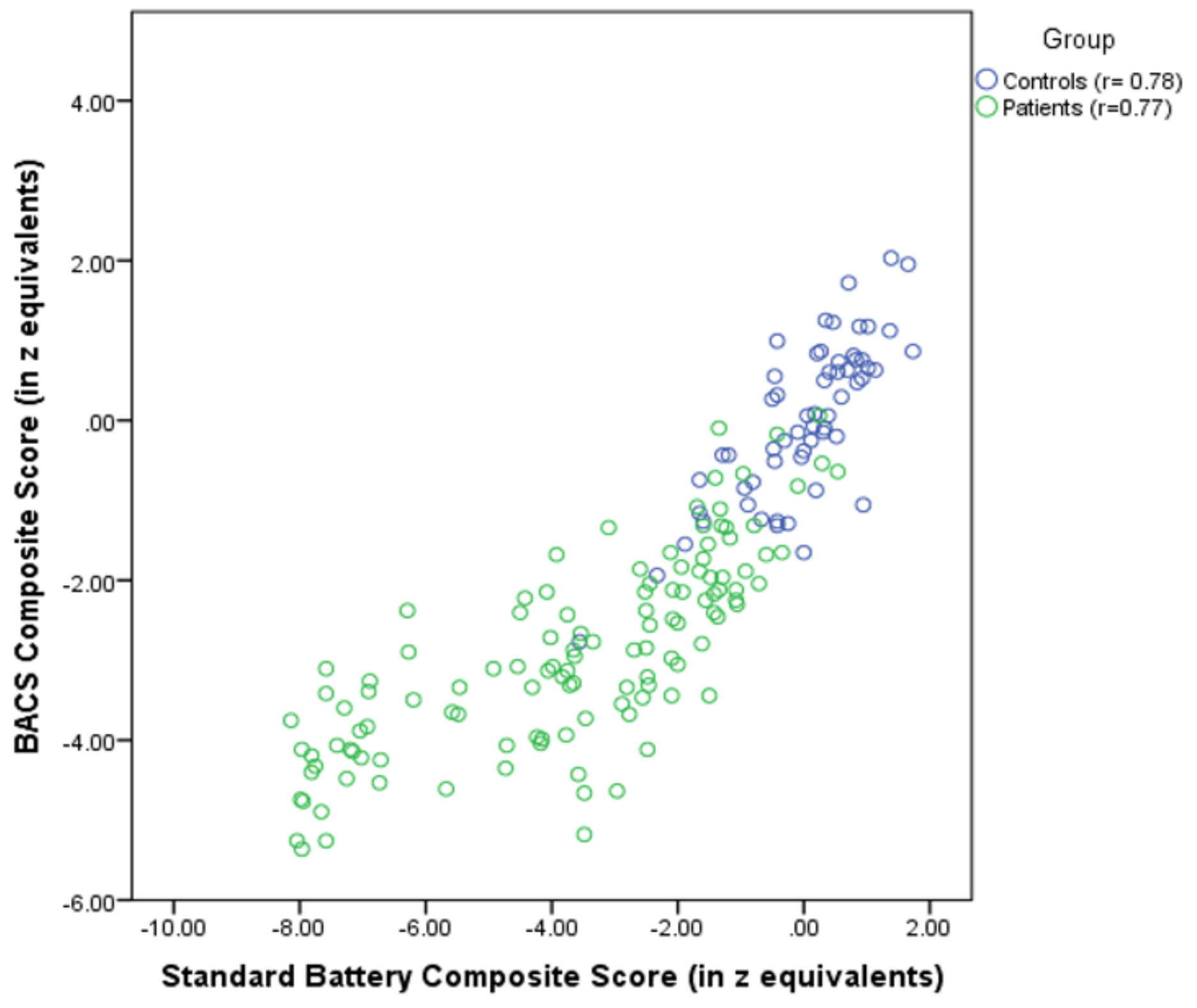

Figure 3

Scatterplots of BACS and standard battery composite scores for patients and controls. 


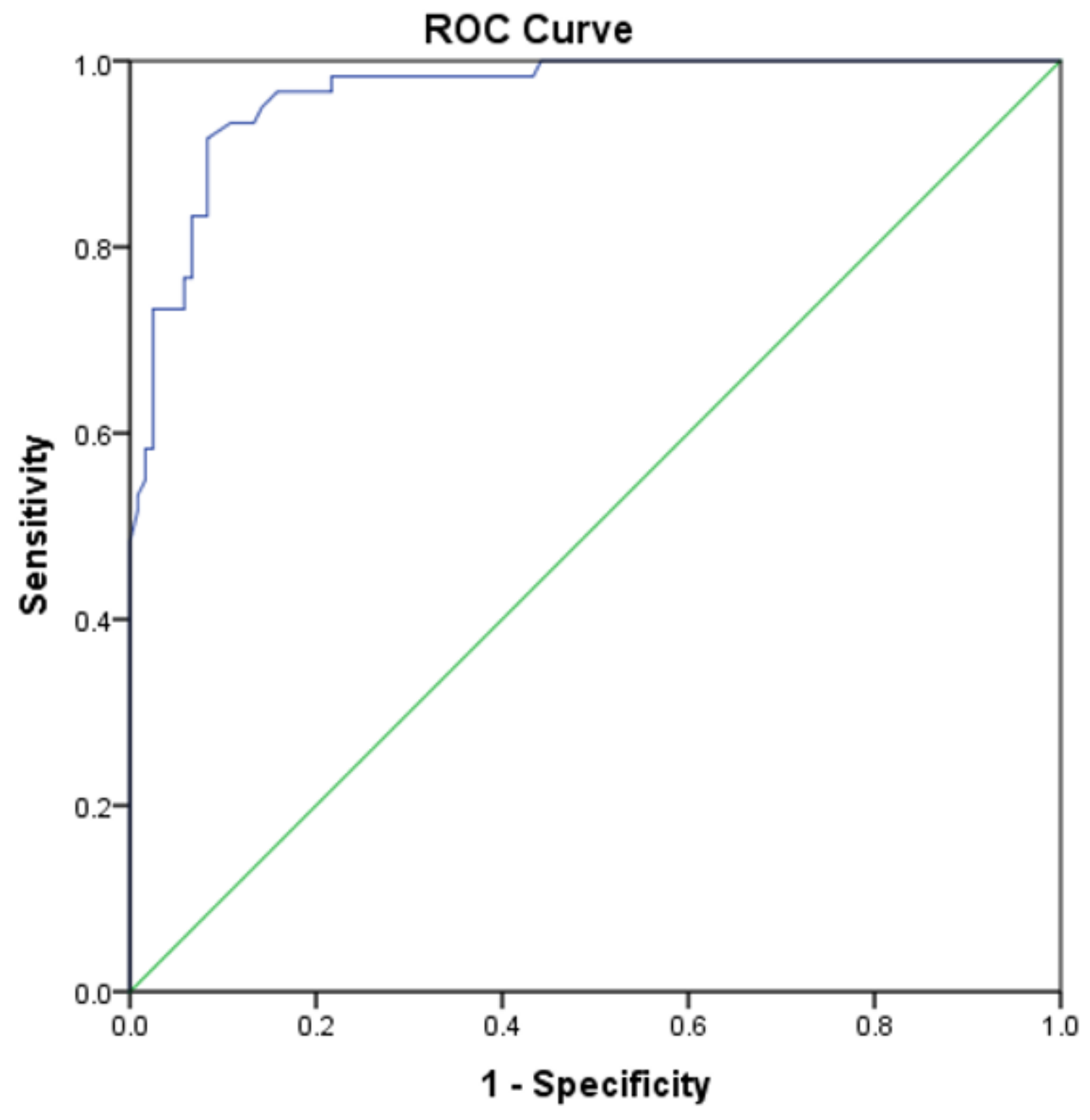

Figure 4

ROC curve of the BACS for the predicted probability for being either identified as a patient or a healthy control. Area under the curve $=.96[.94-0.99](P<.001)$; at value $=163(z$ score value $=-1.51), \mathrm{Se}=93.3 \%$ and $\mathrm{Sp}=86.7 \%$. 